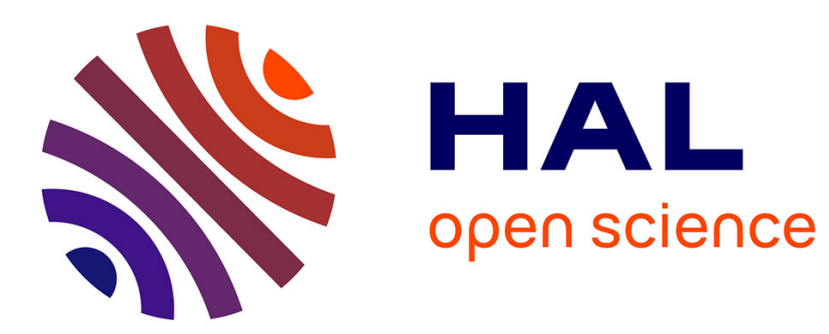

\title{
Électro-ultrafiltration du lait sur membrane minérale: étude des débits et du colmatage
}

B Hugodot, A Bouziane, Mn Zidoune, B Tarodo de La Fuente

\section{To cite this version:}

B Hugodot, A Bouziane, Mn Zidoune, B Tarodo de La Fuente. Électro-ultrafiltration du lait sur membrane minérale: étude des débits et du colmatage. Le Lait, 1991, 71 (6), pp.645-659. hal00929273

\section{HAL Id: hal-00929273 \\ https://hal.science/hal-00929273}

Submitted on 1 Jan 1991

HAL is a multi-disciplinary open access archive for the deposit and dissemination of scientific research documents, whether they are published or not. The documents may come from teaching and research institutions in France or abroad, or from public or private research centers.
L'archive ouverte pluridisciplinaire HAL, est destinée au dépôt et à la diffusion de documents scientifiques de niveau recherche, publiés ou non, émanant des établissements d'enseignement et de recherche français ou étrangers, des laboratoires publics ou privés. 


\title{
Électro-ultrafiltration du lait sur membrane minérale : étude des débits et du colmatage
}

\author{
B Hugodot, A Bouziane, MN Zidoune, B Tarodo de la Fuente
}

USTL, Laboratoire de technologie alimentaire, 34095 Montpellier Cedex 5, France

(Reçu le 3 avril 1990; accepté le 12 juillet 1991)

\begin{abstract}
Résumé - Des essais d'ultrafiltration de lait écrémé reconstitué ont été réalisés sur membrane d'alumine tubulaire, en présence d'un champ électrique transmembranaire, en statique et en dynamique. Par une méthode de réalisation et d'extraction du dépôt présentée ici, nous avons pu montrer qu'en statique, un champ électrique de $300 \mathrm{~V} \cdot \mathrm{m}^{-1}$ correctement orienté ralentit la formation du dépôt. En dynamique, un champ de $100 \mathrm{~V} \cdot \mathrm{m}^{-1}$ établi avant l'introduction du lait apporte une diminution du colmatage de $30 \%$, accompagnée d'une nette augmentation du débit. Cet effet peut atteindre $50 \%$ à $200 \mathrm{~V} \cdot \mathrm{m}^{-1}$. Cette méthode est limitée par le phénomène d'électrolyse, devenant important, dans nos conditions opératoires, à $400 \mathrm{~V} \cdot \mathrm{m}^{-1}$. Les dosages du calcium et des matières azotées totales et l'analyse électrophorétique des protéines des différentes fractions du dépôt permettent de proposer un mécanisme de formation de ce dépôt en statique et en dynamique ainsi qu'un mode d'action du champ sur celui-ci par polarisation de ses molécules.
\end{abstract}

lait / ultrafiltration / champ électrique / colmatage

Summary - Effect of an electric field on the ultrafiltration of milk. Ultrafiltration of reconstituted skim-milk was performed on tubular alumina membranes in the presence of a transmembrane electric field under static and dynamic conditions. By a method for deposit formation and extraction described here, we distinguished 3 different and independent layers in the deposit. Furthermore, we showed that a correctly oriented field of $300 \mathrm{~V} \cdot \mathrm{m}^{-1}$ could slow down the fouling. Under dynamic conditions when a field of $100 \mathrm{~V} \cdot \mathrm{m}^{-1}$ was applied before introduction of the milk, we observed a $30 \%$ decrease in clogging and a marked increase in the flux rate. At $200 \mathrm{~V} \cdot \mathrm{m}^{-1}$ this effect reached $50 \%$. One limitation of the method was electrolysis of the medium; this phenomenon became important at $400 \mathrm{~V} \cdot \mathrm{m}^{-1}$ under our working conditions and led to coagulation of the milk at the cathode. Based on the results of assays of calcium and total nitrogen and electrophoretic analysis of the proteins in the different fractions of the deposit, we propose a mechanism for deposit formation under static and dynamic conditions as well as a mode of action of the electric field involving polarization of molecules in the deposit.

milk / ultrafiltration / electric field / fouling 


\section{INTRODUCTION}

Le colmatage des membranes représente le problème le plus important de l'ultrafiltration et son facteur limitant, en provoquant de fortes chutes du débit d'ultrafiltrat au cours du temps et des modifications de sa composition.

Ce colmatage se produit principalement à 2 niveaux :

- à l'intérieur et à la surface de la couche filtrante, dépôt de macromolécules ou de matières en suspension, adsorbées plus ou moins fortement sur le matériau poreux selon les propriétés de surface des uns et des autres : c'est le dépôt dit «irréversible» car il ne s'élimine pas dans les conditions proches de celles de la filtration, et qui joue le rôle de véritable membrane d'ultrafiltration;

- près de la surface, dans la phase liquide, accumulation de molécules de solutés résultant d'un équilibre convectiondiffusion : c'est la couche de polarisation de concentration, "réversible", et dont l'épaisseur diminue le débit d'ultrafiltration, comme on le verra plus loin.

Parmi les nombreux travaux réalisés dans le domaine des techniques séparatives depuis plus de 10 ans, un grand nombre porte sur la diminution de ce colmatage (Bennasar, 1984; Taddei et al, 1988; Tarodo de la Fuente et al, 1989).

A cette fin, différentes techniques peuvent être utilisées selon l'appareillage employé, le produit traité et les buts recherchés.

Le débit d'ultrafiltrat $J$ est classiquement donné par l'équation générale :

$$
J=\frac{\Delta P}{\mu R_{T}}
$$

où $\Delta \mathrm{P}=$ différence de pression transmembranaire, $\mu=$ viscosité dynamique du filtrat, $R_{T}=$ résistance membranaire totale.

On peut l'améliorer en jouant sur les conditions opératoires :

- en augmentant la pression : il lui est proportionnel jusqu'à quelques bars;

- en diminuant la viscosité par une élévation de la température : celle-ci est limitée par la fragilité du produit :

- en diminuant $R_{T}$ : ce terme peut s'exprimer par la somme

$$
R_{\mathrm{T}}=R_{\mathrm{m}}+R_{\mathrm{d}}
$$

$R_{\mathrm{m}}$ étant la résistance de la membrane elle-même et $R_{d}$ celle du dépôt.

$R_{\mathrm{m}}$, caractéristique de la membrane, et considéré généralement comme intangible, peut être modifié par le conditionnement de la membrane, résultant de la dernière phase du nettoyage de celle-ci. Pour les membranes d'alumine, notamment, un conditionnement alcalin est préférable pour traiter des solutions minérales (pour une filtration stérilisante par exemple), mais un conditionnement acide donne de meilleurs débits avec des solutions protéiques (Zidoune, 1983).

$R_{\mathrm{d}}$, enfin, peut être diminué par une limitation de l'épaisseur du dépôt, que l'on peut obtenir en augmentant les forces de cisaillement le long de la paroi :

- augmentation de la turbulence par accroissement de la vitesse de circulation tangentielle, diminution de la section de passage, présence de reliefs tracés sur la membrane;

- introduction de promoteurs de turbulence entraînés avec le liquide ou utilisés en lit fluidisé.

Milisic et Bersillon (1986) ont comparé certaines de ces techniques et ont trouvé 
que la méthode de filtration à débit pulsé apportait également des améliorations.

On peut aussi diminuer l'épaisseur du dépôt par la limitation de sa formation en éloignant de la paroi les substances colmatantes.

L'utilisation d'un champ électrique pour des liquides contenant des substances chargées électriquement, ou pouvant l'être, peut être une solution.

De très nombreuses recherches ont été réalisées sur l'utilisation des propriétés électrochimiques des membranes et des solutés, en présence ou non de champ électrique.

Citons d'abord quelques exemples en électrodialyse, ou dialyse en présence d'un champ électrique, qui permet de concentrer les ions et molécules chargées entre des membranes échangeuses d'ions anioniques et cationiques en présence d'un champ électrique : méthode très utilisée pour la désionisation de l'eau de mer ou de l'eau courante (Vallot, 1988), mais aussi la désacidification des moûts de raisin (Escudier et al, 1988), la reconcentration d'acides minéraux (Cohen et al, 1988) ou d'acides aminés (Takahashi \& Nakayasu, 1987), la déminéralisation du lactosérum (Letord, 1986), etc.

Ahlgreen (1980) propose de combiner l'électrodialyse à l'ultrafiltration avec un appareillage breveté comportant 2 membranes d'ultrafiltration et 2 couples de membranes échangeuses d'ions, le tout traversé par un champ électrique. Appliqué au lactosérum, ce procédé permet d'obtenir un concentré de protéines et un concentré de lactose, tous deux fortement déminéralisés. Radovitch et Sparks (1980) ont, eux, combiné l'électrodialyse à l'électro-ultrafiltration frontale.

Quant à l'électrofiltration, Wakeman (1986) en a rappelé le principe : "electrofiltration = microfiltration + electro- phoresis"; Krishnaswamy et Klinkowski (1986) en ont présenté une revue assez complète; Turkson (1987) l'a appliquée à la sérumalbumine bovine sur des membranes rotatives, lui associant ainsi la centrifugation.

Visvanathan et Ben Aïm (1986) ont exposé la théorie de l'électromicrofiltration tangentielle et l'ont appliquée au bleu dextrane sur membrane plane et Vivoni et al (1986) ont présenté les calculs théoriques concernant l'électro-ultrafiltration et montré qu'avec une solution de sérumalbumine bovine on peut pratiquement annuler, par un champ électrique, tous les effets de la polarisation de concentration.

Certains utilisent des membranes chargées seules (Kimura et Tamano, 1986), d'autres des membranes conductrices en présence d'un champ électrique (Guizard et al, 1989).

Dans notre laboratoire, l'électroultrafiltration a été associée avec succès à la présence de promoteurs de turbulence en lit fluidisé (Rios et al, 1986) et divers essais ont été réalisés en électroultrafiltration simple sur des solutions modèles de protéines, comme par exemple la gélatine, pure (Rios et al, 1986; Freund, 1989) ou en mélange avec l'hémoglobine, et il nous a paru utile de franchir une nouvelle étape en travaillant directement sur le lait.

Sur ce produit, en effet, on pratique de plus en plus couramment une ultrafiltration tangentielle en vue d'en concentrer les protéines, avant son traitement en fromagerie, par exemple.

Les matières colmatantes sont alors essentiellement les protéines lactiques dans leur ensemble - y compris celles du lactosérum, qui sont également retenues par ce procédé - avec la participation de sels minéraux. 
Or, au pH normal du lait $(6,6-6,8)$, la plupart des protéines sont chargées négativement (Swaisgood, 1982) : un champ électrique transmembranaire correctement appliqué devrait les éloigner de la surface filtrante.

Bien que les conditions hydrodynamiques soient très différentes entre les expérimentations en statique (pression très faible et vitesse de circulation nulle) et en dynamique et entraînent une cinétique de formation et une composition du dépôt propres à chaque cas, les travaux antérieurs de Vétier et al (1986) ont montré que la composition qualitative des fractions réversible et irréversible du colmatage étaient semblables. Nous avons alors commencé par une étude en statique, sur un appareillage de laboratoire, suivie d'une expérimentation en dynamique sur pilote. Les résultats analytiques obtenus nous ont permis d'envisager un mécanisme de formation du dépôt, et un mode d'action du champ électrique.

\section{MATÉRIEL ET MÉTHODES}

\section{Expérimentations en statique}

Nous avons utilisé des membranes d'alumine $(99,9 \%$ d'alumine $\alpha)$ tubulaires de diamètre intérieur/extérieur de $15 / 19 \mathrm{~mm}$, à surface filtrante interne de $0,2 \mu \mathrm{m}$ de diamètre moyen de pores, fournies par la Société des céramiques techniques (SCT, 65460 Bazet). Nous avons débité les membranes initiales de $750 \mathrm{~mm}$ de long en éléments de $75 \mathrm{~mm}$ et de $32 \times 10^{-4} \mathrm{~m}^{2}$ de surface utile.

Nous avons toujours travaillé avec des membranes neuves, n'ayant subi aucun rinçage ni conditionnement, et à une température de $20^{\circ} \mathrm{C}$.

\section{Le lait}

Le lait a été reconstitué dans de l'eau permutée à partir de lait écrémé en poudre provenant de SA Gilap, 29150 Chateaulin et maintenu $24 \mathrm{~h}$ en chambre froide $\left(4-6^{\circ} \mathrm{C}\right)$ avant utilisation, afin de permettre l'établissement des équilibres salins et micellaires.

Sa composition en grammes par litre est la suivante :

- matière sèche totale : 96,8 ;

- matières grasses : $<0,5$;

- matières azotées totales : $33,6($ MAT $=\mathrm{N} x$

6,38);

- matières azotées non protéiques : $2,16(\mathrm{~N} \times$

6,38);

- calcium total : 1,3 (Ca/MAT =0,039);

- phosphore total : 1,0;

$-\mathrm{pH}=6,70 \pm 0,05$ à $20^{\circ} \mathrm{C}$.

\section{Méthode de réalisation du dépôt}

Celle-ci utilise le montage de la figure 1, dans lequel la membrane, munie d'un manchon absorbant et le cas échéant, des électrodes, est emplie de lait avant d'être raccordée à la burette graduée, elle aussi emplie de lait. Le manchon absorbant est destiné à éviter l'évaporation du filtrat à la surface du poreux, surtout lors de longs essais. Les électrodes ne sont installées que lors des essais avec champ électrique; leur distance est de $10 \pm 0,5 \mathrm{~mm}$. La hauteur moyenne de la colonne de lait est de $0,75 \mathrm{~m}$; la pression au niveau de la membrane est donc de $7500 \pm 350 \mathrm{~Pa}$. Les débits, avec le lait, sont trop faibles pour être exploitables. La quantité minime qui traverse la membrane est retenue par le manchon et n'a pas été mesurée.

À la fin de l'essai, on ferme le robinet de la burette et on en sépare la membrane afin d'étudier le dépôt formé.

\section{Extraction du dépôt}

Pour extraire le dépôt, on vide la membrane par aspiration à la pipette en surface afin d'éviter 


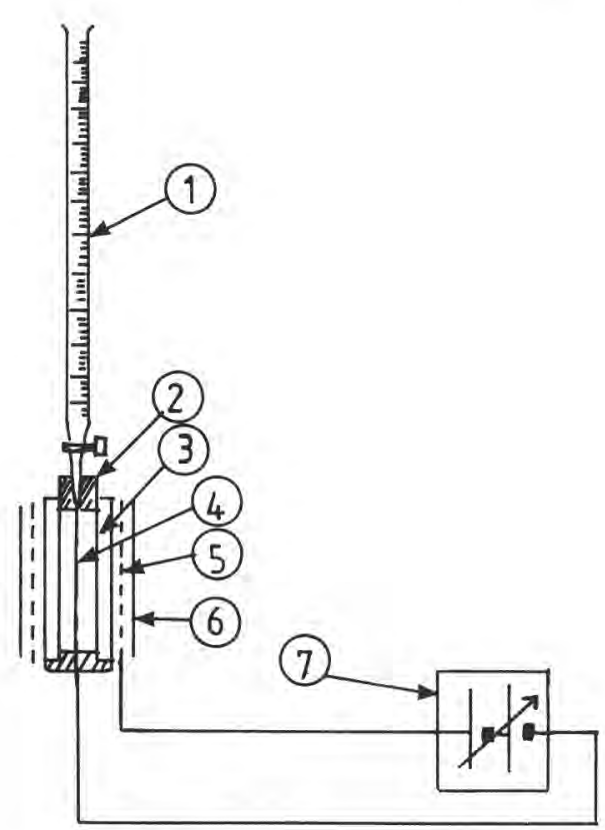

Fig 1. Montage pour l'étude du colmatage en statique : 1 = burette graduée emplie de lait; 2 = bouchon de caoutchouc; $3=$ membrane emplie de lait; 4 = anode (fil de Pt axial); $5=$ cathode (grille inox); 6 = manchon absorbant; 7 = potentiostat.

Set-ups for the study of fouling under static conditions : 1 = graduated burette filled with milk; 2 = caoutchouc tip; 3 = membrane filled with milk; 4 = anode (Pt axial wire); 5 = cathode (stainless steel grid); $6=$ absorbing cylinder; $7=$ potentiostat.

tout mouvement de liquide le long des parois, on ôte le bouchon et on le rince, on tamponne le bas de la membrane pour en éliminer le lait restant, on rebouche puis on remplit d'eau permutée en limitant au maximum, pour la même raison, les courants de convection.

Après 2 min de contact, on récupère, en opérant comme précédemment, la première solution de rinçage SR1 et l'on répète l'opération jusqu'à l'obtention d'une fraction limpide. Les différentes fractions SR sont réunies pour l'ana- lyse; elles constituent la partie "réversible" du colmatage, comprenant la partie de la couche de polarisation adjacente au dépôt et la partie de ce dépôt la moins fixée. Qualitativement, cette fraction contient les différents constituants du lait.

La membrane est ensuite traitée aux ultrasons pendant $30 \mathrm{~min}$ dans un tube métallique contenant $30 \mathrm{ml}$ d'eau permutée pour donner la fraction US, qui constitue la partie "irréversible" du colmatage.

Enfin, elle est broyée et la poudre formée est analysée : on obtient le résidu membranaire RM.

Sur ces fractions, on dose les matières azotées totales (MAT) par la méthode de Kjeldahl sur appareil Büchi 322/342 et le calcium par absorption atomique sur appareil Varian Techtron AA6.

Comme les protéines sont de loin les principales substances colmatantes (Cheryan et Merin, 1980; Gernedel, 1980), on évaluera le dépôt, tout au long de cette étude, par sa concentration en MAT. Les valeurs données (fig 4) correspondent aux $32 \times 10^{-4} \mathrm{~m}^{2}$ de la surface membranaire utile en statique, à la même surface en dynamique.

Nous avons également analysé par électrophorèse sur gel de polyacrylamide $(12,5 \%$ d'acrylamide) en milieu SDS (méthode de Laemmli modifiée par Zidoune, 1983) la composition en protéines de chacune des fractions SR et US de quelques essais : développement sur appareil Elcam 84 Camag, révélation au bleu de Coomassie puis tracé densitométrique des spectres sur l'appareil Electrophoresis scanner Camag.

\section{Expérimentations en dynamique}

Les membranes ont les mêmes caractéristiques que pour les essais en statique, mais leur longueur est de $0,15 \mathrm{~m}$ et leur surface utile de $70 \mathrm{x}$ $10^{-4} \mathrm{~m}^{2}$.

L'installation pilote est représentée figure 2.

Le lait provient du même lot et a subi le même conditionnement que celui des essais statiques.

Les conditions opératoires choisies sont les suivantes : 


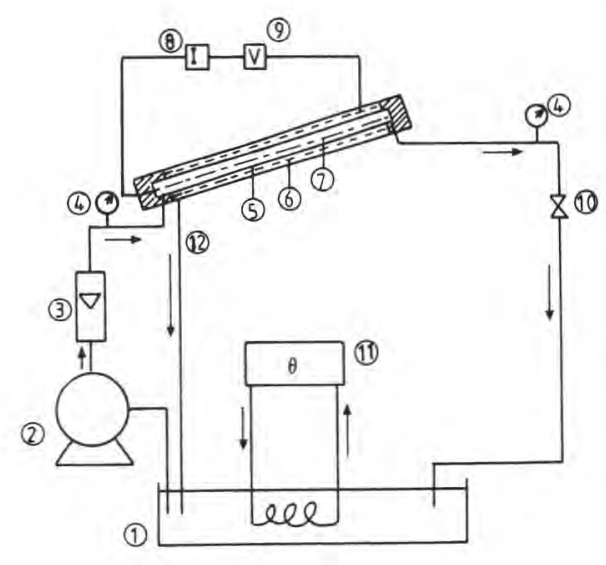

Fig 2. Schéma de l'installation pilote : 1 = bac d'alimentation; 2 = pompe d'alimentation; 3 = débitmètre; $4=$ manomètre; $5=$ membrane; $6=$ cathode (grille inox); $7=$ anode axiale (fil inox); $8=$ ampèremètre; $9=$ potentiostat; $10=$ vanne de pression; 11 = cryostat; $12=$ recyclage de l'ultrafiltrat.

Schematic diagram of the pilot apparatus : $1=$ feeding tank; 2 = feeding pump; 3 = fluxmeter; $4=$ manometer $; 5=$ membrane; 6 : cathode (stainless steel grid); $7=$ axial anode (stainless steel wire); 8 = amperemeter; 9 = potentiostat; $10=$ pressure paddle $; 11=$ cryostat $; 12=$ ultrafiltrate feed back.

- pression transmembranaire moyenne : 2 bar;

- vitesse tangentielle: $2 \mathrm{~m} \cdot \mathrm{s}^{-1}$;

- température : $20^{\circ} \mathrm{C}$;

- ultrafiltrat recyclé en totalité.

La durée des ultrafiltrations sans champ et avec champ préétabli était de $2 \mathrm{~h}$ avec recyclage total du filtrat. Pour les essais avec champ postétabli, nous avons attendu $2 \mathrm{~h}$ avant la mise sous tension, afin d'être en débit stabilisé et d'avoir un maximum de dépôt.

Les valeurs données ont été ramenées à une surface membranaire de $32 \times 10^{-4} \mathrm{~m}^{2}$, comme pour les essais en statique.

L'extraction du dépôt et les analyses se font comme en statique, en tenant compte des dimensions différentes des membranes.

\section{RÉSULTATS}

Les résultats qui suivent sont généralement la moyenne de 5 essais sans champ, de 2 essais avec champ.

Les incertitudes relatives affectant ces résultats sont de l'ordre de $5 \%$ pour les MAT des fractions SR, de $10 \%$ pour les US et le $\mathrm{Ca}$ (dans les rapports $\mathrm{Ca} / \mathrm{MAT}$ ) et de 5 à $20 \%$ de la plus forte à la plus faible concentration pour les électrophorèses.

\section{En statique}

\section{Caractérisation du montage électrique}

Afin de caractériser le système et d'évaluer le champ maximal imposable, nous avons tracé la courbe intensité $=f($ potentiel $)$, l'anode étant l'électrode axiale en fil de platine (fig 3).

Au départ, avant d'allumer le potentiostat, on observe dans le montage une légère polarisation positive qui se traduit au niveau de la courbe par un décalage en abscisses de $100 \mathrm{~V} \cdot \mathrm{m}^{-1}$. Ce phénomène est probablement dû à un effet de pile entre le platine de l'électrode axiale et l'acier inoxydable de la grille périphérique, éventuellement modifié par le rapport des surfaces des 2 électrodes et par une peu probable différence de potentiel électrocapillaire créée par le passage des électrons à travers la membrane et son support poreux.

La courbe présente, de 0 à $200 \mathrm{~V} \cdot \mathrm{m}^{-1}$, une partie pratiquement linéaire de pente voisine de $0,01 \mathrm{~mA} / \cdot \mathrm{m}^{-1}$. Au-delà, elle s'infléchit vers le haut.

À partir de $450 \mathrm{~V} \cdot \mathrm{m}^{-1}$, l'intensité est instable et croît continuellement à partir de sa valeur de départ tandis que se produit une importante électrolyse dans le tube fil- 


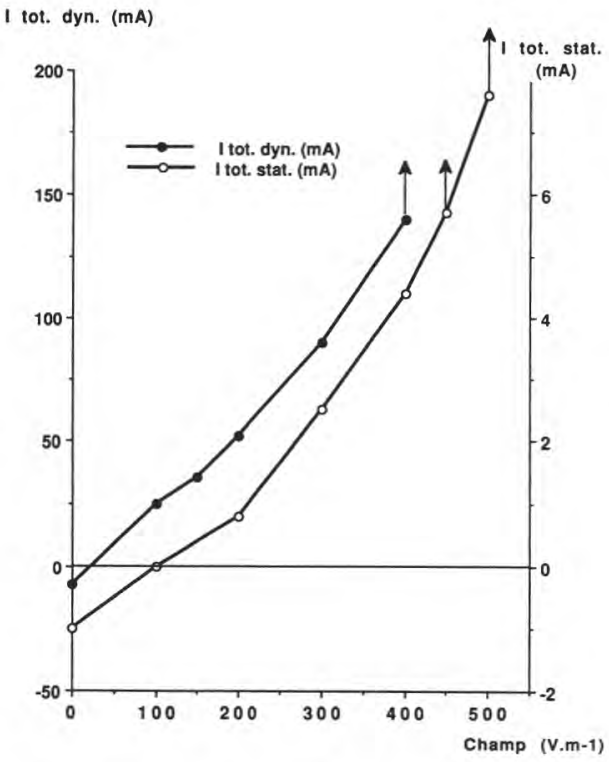

Fig 3. Courbes $I$ (intensité totale débitée) $=\mathrm{f}$ $\left(V \cdot m^{-1}\right)$ en statique et en dynamique. Les flèches indiquent l'instabilité de I.

I (total intensity) $=f\left(V \cdot m^{-1}\right)$ under static and $d y$ namic conditions. The arrows indicate the instability of $\mathrm{I}$.

trant, qui donne lieu à un dégagement gazeux notable et à la formation d'un dépôt autour du fil de platine. En conséquence, nous avons pris une valeur maximale de $400 \mathrm{~V} \cdot \mathrm{m}^{-1}$ pour les essais en statique. $\grave{A}$ cette valeur, le courant total débité, de $4,4 \mathrm{~mA}$, provoque une très faible électrolyse accompagnée d'une formation de mousse de quelques $\mathrm{mm}$ d'épaisseur.

\section{Étude des dépôts}

Le champ appliqué était de $400 \mathrm{~V} \cdot \mathrm{m}^{-1}$. Les essais avec un champ de $300 \mathrm{~V} \cdot \mathrm{m}^{-1}$ ont donné les mêmes résultats que sans champ.

\section{MAT (fig 4)}

En statique, l'effet du champ électrique est pratiquement le même, qu'il soit établi avant le début de l'essai (champ préétabli) ou après (champ postétabli) : nous le représentons par une seule courbe moyenne.

- la fraction RM est représentée par une seule série de points car sa valeur reste pratiquement constante $(6 \pm 1 \mathrm{mg})$, avec ou sans champ.

- La fraction US montre une croissance régulière dans le temps à partir de $t=10$

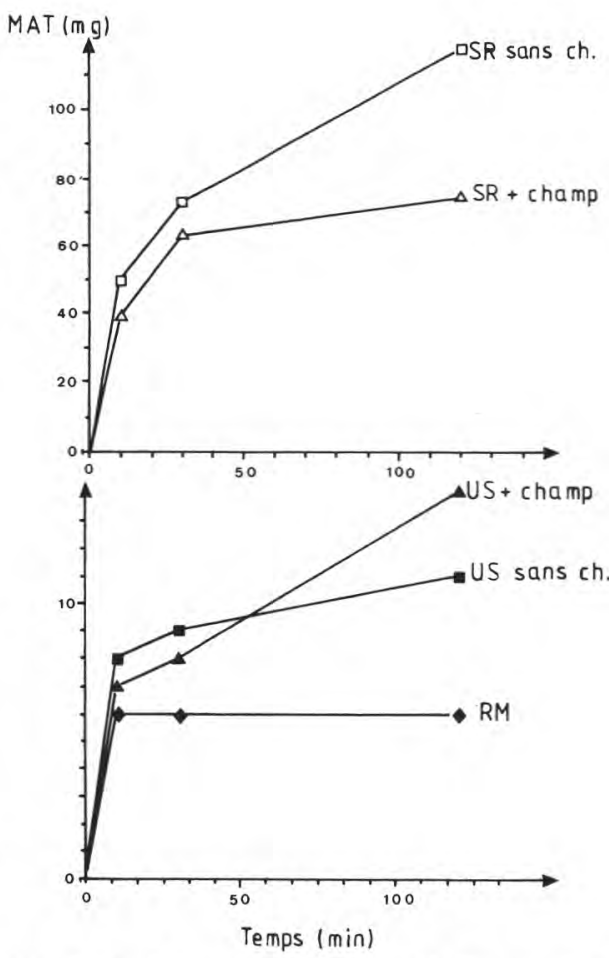

Fig 4. Évolution des MAT des dépôts en statique en fonction du temps. Champ électrique $400 \mathrm{~V} \cdot \mathrm{m}^{-1}$.

Effect of assay duration on total nitrogen content (MAT) under static conditions at 400 $V \cdot m^{-1}$. 
min, de l'ordre de 1,5 mg•h-1 sans champ, de $3 \mathrm{mg}^{\circ} \mathrm{h}^{-1}$ avec champ.

- Pour la fraction SR, le champ paraît ralentir - voire stabiliser - l'accumulation des matières colmatantes, qui semble très rapide dès la mise en contact du lait et de la membrane.

\section{Rapport Ca/MAT}

La figure 5 montre la différence de composition entre les fractions US et SR : le rapport $\mathrm{Ca} / \mathrm{MAT}$ des premières reste presque toujours supérieur à celui du lait, alors que pour les secondes, il lui est toujours inférieur.

Dans la fraction US, ce rapport double presque, sans champ, de $t=10 \min$ à $t=$ $2 \mathrm{~h}$. En présence de champ, au contraire, les fortes concentrations en $\mathrm{Ca}$ de la pre-

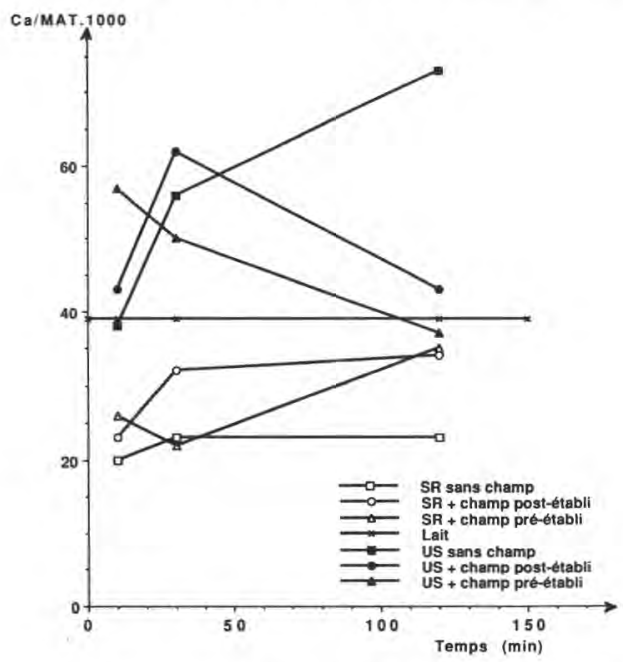

Fig 5. Rapports Ca/MAT en fonction du temps en statique, champ électrique $400 \mathrm{~V} \cdot \mathrm{m}^{-1}$ : SR et US sans champ ou avec champ pré- ou postétabli.

Ratio of Ca/MAT as a function of time under static conditions at $400 \mathrm{~V} \cdot \mathrm{m}^{-1}$. SR and US in the absence of an electric field or in a pre- or postapplied field. mière demi-heure baissent progressivement jusqu'à se retrouver, au bout de $2 \mathrm{~h}$, autour de celles du lait.

Dans la fraction SR, le rapport Ca/MAT est faible et pratiquement constant, sans champ. Celui-ci a pour effet d'augmenter ce rapport jusqu'à une valeur proche, au bout de $2 \mathrm{~h}$, de celle du lait de départ.

\section{Électrophorèses}

Les profils électrophorétiques obtenus au densitomètre permettent de distinguer 4 massifs contenant respectivement l' $\alpha$ lactalbumine $(\alpha, \mathrm{L} a)$, la $\beta$-lactoglobuline $(\beta \mathrm{Lg})$, la caséine totale (Cas) et la sérumalbumine (SAB).

La figure 6 traduit en histogrammes, exprimés en \% des protéines totales, les proportions des principales fractions (à partir

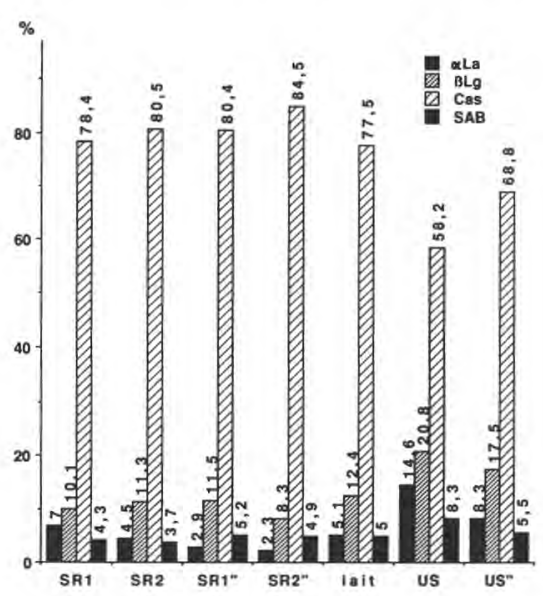

Fig 6. Composition protéique (en $\%$ des protéines totales) du lait et de fractions de dépôts (contacts $30 \mathrm{~min}$ ) : représentation par histogrammes. SR1, SR2, US = sans champ; SR1", SR2", US" = champ préétabli.

Bar graph representing the proteic composition (in \% of total proteins) of milk and of the deposit fractions (30-min contact time). SR1, SR2, US : in the absence of an electric field; SR1", SR2", US" : pre-applied field. 
de la $3^{e}$ fraction SR, les teneurs sont trop faibles pour donner des résultats significatifs).

Les fractions SR montrent des compositions en protéines semblables à celle du lait initial, variant peu en fonction du champ.

Les fractions US contiennent une proportion plus importante en protéines sériques par rapport aux caséines, notamment en $\alpha$-lactalbumine et en $\beta$-lactoglobuline, cette différence étant apparemment réduite en présence du champ.

\section{En dynamique}

\section{Courbe intensité/potentiel (fig 3)}

Ici, les 2 électrodes sont en acier inoxydable, et le potentiel de polarisation ne se traduit plus que par un décalage en abscisses de la courbe de $20 \mathrm{~V} \cdot \mathrm{m}^{-1}$.

Celle-ci montre, comme en statique, une partie linéaire de 0 à $200 \mathrm{~V} \cdot \mathrm{m}^{-1}$, mais

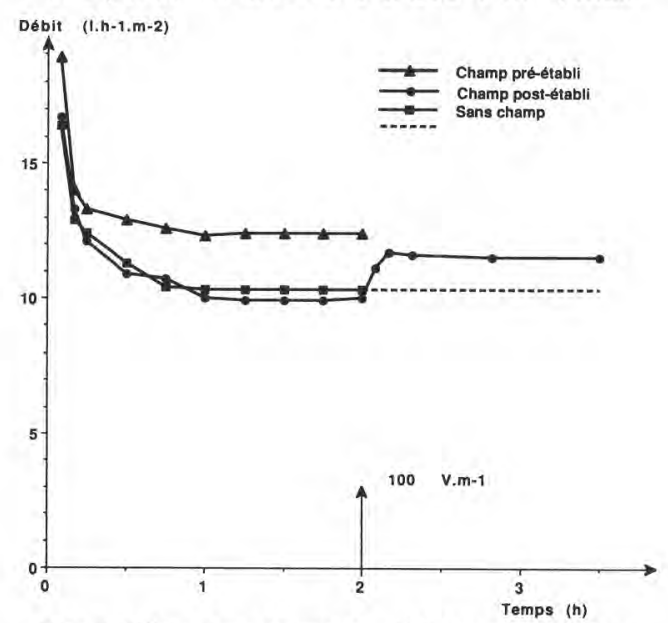

Fig 7. Effet d'un champ de $100 \mathrm{~V} \cdot \mathrm{m}^{-1}$ sur l'évolution dans le temps du débit d'ultrafiltrat.

Effect of assay duration at $100 \mathrm{~V} \cdot \mathrm{m}^{-1}$ on the ultrafiltrate flux rate. avec une pente environ 30 fois supérieure, due à l'augmentation du débit de perméation et au passage d'ions et de molécules chargées.

L'intensité commence à dériver avec début d'électrolyse importante dès 400 $v \cdot m^{-1}$.

\section{Effets de champs électriques}

\section{Sur les performances}

La figure 7 montre que l'imposition d'un champ préétabli apporte une amélioration du débit d'environ $26 \%$ et que, si ce champ est imposé après $2 \mathrm{~h}$ de fonctionnement, cette amélioration est de l'ordre de $15 \%$ et se fait progressivement, en $10 \mathrm{~min}$ environ.

Avec des champs supérieurs (fig 8), les débits d'ultrafiltration augmentent à chaque accroissement du champ.

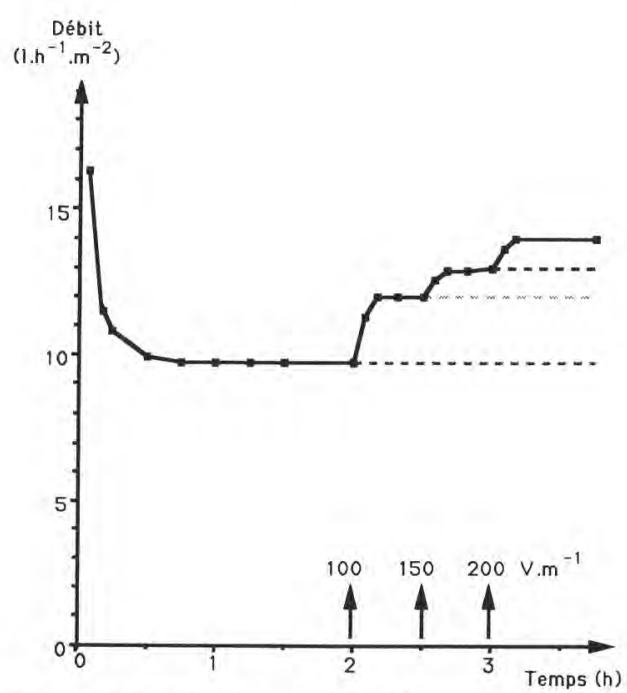

Fig 8. Effet de champs électriques croissants sur le débit d'ultrafiltrat.

Effect of increasing electric fields on the ultrafiltrate flux rate. 
Pour un champ de $400 \mathrm{~V} \cdot \mathrm{m}^{-1}$ postétabli d'un coup au bout de $2 \mathrm{~h}$, le débit est augmenté de $35 \%$, donc moins qu'avec les montées progressives représentées dans la figure 8 , qui totalisent une amélioration de $44 \%$ à $200 \mathrm{~V} \cdot \mathrm{m}^{-1}$. Le phénomène d'électrolyse devient alors très important avec une forte production de mousse, une rapide corrosion de l'électrode axiale et la formation sur celle-ci d'un épais dépôt blanc et brunâtre, adhérant fortement et très riche en azote et en fer.

\section{Sur les dépôts}

Les résultats des analyses effectuées sur les dépôts après $2 \mathrm{~h}$ d'ultrafiltration sont rassemblés dans la figure 9 .

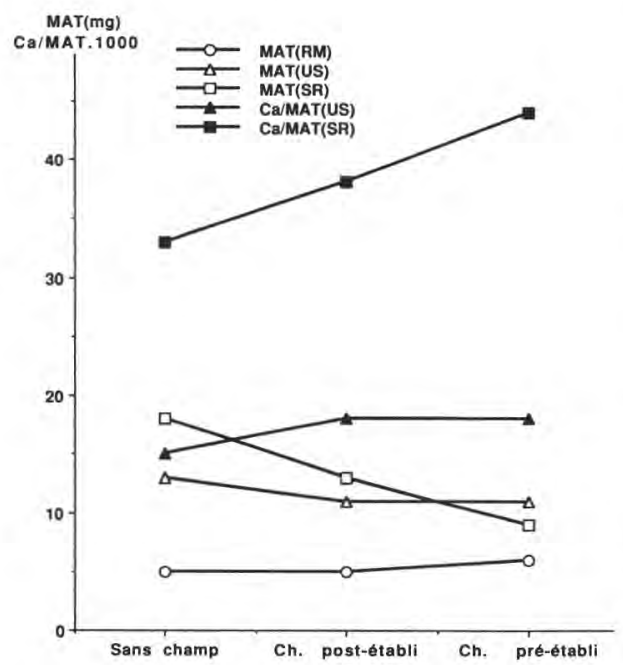

Fig 9. Composition des couches de dépôt en dynamique. Champ de $100 \mathrm{~V} \cdot \mathrm{m}^{-1}$. Valeurs ramenées à une surface membranaire de $3210^{-4}$ $\mathrm{m}^{2}$.

Composition of the deposit layers obtained under dynamic conditions at $100 \mathrm{~V} \cdot \mathrm{m}^{-1}$. Values for a membrane area of $3210^{-4} \mathrm{~m}^{2}$.
Les dosages de MAT à $t=2 \mathrm{~h}$ montrent, avec un champ de $100 \mathrm{~V} \cdot \mathrm{m}^{-1}$, que les meilleurs résultats sont obtenus avec champ préétabli, un champ postétabli présentant une efficacité nettement moindre. La teneur en MAT reste faible et voisine de $5 \mathrm{mg}$ dans la fraction RM, paraît diminuer légèrement (de 13 à $11 \mathrm{mg}$ ) dans la fraction US et chute fortement (de 120 à 80 $\mathrm{mg}$ ) dans la fraction SR.

Le rapport Ca/MAT passe de $3310^{-3}$ à $4410^{-3}$ dans les fractions SR et de $1510^{-3}$ à $1810^{-3}$ dans les fractions US.

\section{DISCUSSION}

\section{Colmatage en statique}

Le colmatage interne RM est une fraction très faible du dépôt. S'il n'était qu'un résidu d'ultrafiltrat dont resterait imbibé le corps de la membrane à la fin de l'essai, il serait facilement extractible. S'il était dû à une adsorption de constituants de l'ultrafiltrat, sa masse augmenterait dans le temps. Or, RM est quasi indépendant des conditions de travail et se stabilise pratiquement dès les premières minutes de contact entre le lait et la membrane, donc dès la formation du dépôt d'ultrafiltration.

La fraction US, en statique, augmente avec le temps, alors que RM ne varie pas : ces 2 fractions sont bien indépendantes et la croissance d'US se fait vers l'extérieur de la couche filtrante, alimentée par la couche de polarisation.

Les rapports Ca/MAT des différentes fractions ne peuvent s'expliquer par un relargage de Ca par la membrane. Les propriétés échangeuses de cations de l'alumine sont en effet connues (Helfferich, 1962) et le calcium fixé par celle-ci ne peut être arraché que par lavage acide. 
Sans champ, ces rapports ont des évolutions dans le temps divergentes pour les couches US et SR.

En présence du champ, il paraît se produire une inversion de ces évolutions tendant à rapprocher les rapports correspondant à SR et à US autour de celui du lait, comme si l'effet global du champ consistait en une homogénéisation progressive des fractions.

La diminution par le champ, au cours du temps, du rapport Ca/MAT de la fraction US est à relier à la forte augmentation de la teneur en MAT de cette fraction. Au cours du temps, des matières azotées fixant peu le calcium s'accumuleraient, tandis que cet élément serait entraîné, par migration électrophorétique, au-delà de la couche protéique, vers la cathode.

Les électrophorèses, enfin, réalisées sur des échantillons prélevés 30 min après la mise en conditions en statique, donnent, sans champ, pour les fractions SR, des proportions de protéines proches de celles du lait initial tandis que dans les fractions US se produit un enrichissement relatif en protéines sériques, surtout en $\alpha$ lactalbumine et $\beta$-lactoglobuline, aux dépens de la caséine.

Cette composition des fractions US en l'absence de champ paraît contradictoire avec les valeurs des rapports Ca/MAT analysées ci-dessus. En effet, il est bien connu - et Baumy et Brulé (1988a, b) l'ont encore montré récemment - que la caséine présente des capacités de fixation du calcium largement supérieures à celles des protéines sériques. Par conséquent, l'abaissement de la teneur en caséine de ces fractions, observé en l'absence de champ, devrait s'accompagner d'une chute du rapport $\mathrm{Ca} / \mathrm{MAT}$, si celui-ci ne provenait que du $\mathrm{Ca}$ fixé sur les protéines. La nette augmentation de ce rapport nous amène au contraire à supposer une certaine accu- mulation de sels de calcium non fixés initialement aux protéines - qui représentent $33 \%$ du calcium lactique (Alais, 1984) peut-être par un mécanisme de migration électrophorétique des ions calcium due aux résidus de charges négatives portés par les protéines déposées.

Cette augmentation est certainement grandement facilitée par l'extrême faiblesse - voire la nullité - du débit de filtration, ce qui permet une polarisation de concentration en calcium qui peut, d'une part, provoquer une précipitation de phosphate de calcium et d'autre part, donner lieu à des liaisons additionnelles avec les micelles de caséine déposées, rendant ces protéines moins hydrophiles et moins chargées (Holt, 1982; Green, 1982a, b).

L'effet du champ paraît peu important sur la composition des fractions SR, peu éloignée de celle du lait, tandis que, sur celle des US, il provoque, à $t=30 \mathrm{~min}$, un net rapprochement de la composition protéique du lait par un appauvrissement en protéines sériques. On peut admettre que l'effet du champ soit plus important sur les protéines de faible masse molaire car il s'ajoute à leur plus grande diffusivité.

Quant au calcium, il paraît être décroché progressivement des protéines du dépôt irréversible pour se diriger probablement vers la cathode, ce qui explique la baisse dans le temps du rapport $\mathrm{Ca} / \mathrm{MAT}$ des US.

\section{Filtration en dynamique}

Sur les couches RM et SR, on peut faire les mêmes commentaires qu'en statique.

Pour les fractions US, le rapport $\mathrm{Ca}$ / MAT est faible et augmente peu en présence de champ. Les variations sont donc différentes de ce qu'elles sont en statique; il est probable qu'en dynamique le débit 
important de perméation interdise toute accumulation de sels dans les couches US aussi bien en l'absence qu'en présence de champ électrique.

L'effet de ce dernier s'ajoute à celui des conditions hydrodynamiques pour les fractions SR, alors qu'il est très faible pour les fractions US et nul pour les fractions RM.

Ces résultats nous permettent d'envisager le processus suivant de formation du dépôt :

Dès que le lait se trouve en contact avec la membrane, les protéines isolées ou sous forme de micelles, dispersées dans le sérum par l'agitation thermique et les répulsions électrostatiques, sont entraînées vers la paroi par le débit transmembranaire et le transfert convectif dus aux forces de pression et de capillarité : il se produit immédiatement une accumulation de ces protéines sur la paroi pour former la couche de polarisation et le dépôt, avec, au départ, le passage de quelques molécules et micelles donnant leur léger trouble aux tout premiers $\mathrm{ml}$ de filtrat et la formation du "colmatage interne" RM.

La disparition très rapide du trouble laisse supposer que le dépôt crée très rapidement une barrière au passage des protéines susceptibles d'alimenter RM, transformant la membrane de diamètre de pores de 0,2 $\mu \mathrm{m}$ en une membrane d'ultrafiltration.

Par-dessus, la couche de polarisation se dépose.

Provenant de celle-ci, des micelles de caséine se fixent progressivement sur la couche irréversible, cependant que dans les interstices s'accumulent les protéines sériques, dont la taille est très inférieure à celle des micelles (Gernedel, 1980). Le taux de caséine s'en trouve réduit de 1/3 par rapport à ce qu'il est dans le lait et les couches SR, au profit des protéines sériques, dans l'ordre inverse de leurs masses moléculaires (Alais, 1984) : $\alpha$-lactalbumine ( $M=14200)$ multipliée par 3 , $\beta$-lactoglobuline $(M=36000$ à $\mathrm{pH}<7)$ par 2 , sérumalbumine $(M=69000)$ par 1,5 . En outre, d'après les résultats de Brulé et Fauquant (1981) et le modèle de Schmidt (1982), les ions minéraux (calcium et phosphate en particulier) entraînés avec le filtrat peuvent s'adsorber sur ces protéines, ou précipiter du fait de leur polarisation de concentration (Fox et Mulvihill, 1982). Rendu de plus en plus compact et hydrophobe, le dépôt devient de plus en plus «irréversible» : la couche US s'épaissit au cours du temps.

On peut trouver une confirmation indirecte de cette interprétation dans les résultats obtenus par Vétier et al (1986) avec des laits crus entier et écrémé : dans les dépôts du premier, les micelles protéiques sont plus ou moins dispersées par les globules gras en suspension et le rapport $\mathrm{Ca}$ MAT, indice de la compacité du dépôt, reste toujours inférieur à celui que l'on observe avec le lait écrémé.

En dynamique, l'établissement des couches SR et US est fonction des forces de cisaillement le long de la paroi; l'influence de ces forces est très sensible sur la fraction SR, en l'absence comme en présence de champ électrique, alors qu'elle est pratiquement nulle sur la fraction US, dans les conditions opératoires utilisées. Pour les fractions SR, les phénomènes de convection et de diffusion s'équilibrent pour donner une composition peu différente de celle du lait initial. Ces couches contribuent à la diminution du débit de perméation.

Enfin, l'effet du champ paraît double : c'est d'abord, quantitativement, une réduction, dans la fraction SR, de l'ensemble de la masse protéique, peu ou pas agglomérée, en l'éloignant de la membrane. Ce faisant, il permet une augmentation sensible du débit de filtration. 
En outre, sur US, il paraît opérer une modification de la structure de la couche en diminuant dans le temps son rapport Ca/MAT en statique, en lui permettant de s'enrichir en caséine en dynamique, comme s'il opérait une sorte d'homogénéisation des couches.

Cela fait penser à une orientation progressive dans le sens du champ, donc perpendiculairement à la paroi, des molécules du dépôt chargées ou polarisées par le champ, probablement accompagnée d'une certaine déformation des molécules par allongement. Cette modification de structure des couches amènerait, d'une part, la perte des minéraux le plus faiblement fixés et la baisse du rapport Ca/MAT et d'autre part, une diminution de la compacité de la couche US.

Cette hypothèse est confortée par l'efficacité pratiquement double à tous les niveaux du champ préétabli par rapport au champ postétabli : en effet, il ne s'agit plus pour le champ, dans ce dernier cas, d'éviter ou de ralentir la formation d'un dépôt mais de désagréger et remettre en suspension une partie d'un dépôt déjà formé. En outre, les molécules chargées ou polarisables qui se déposent sur la paroi seraient, avec le premier, déjà polarisées et orientées par rapport au champ.

\section{CONCLUSION}

La méthode mise au point pour la réalisation des dépôts et pour leur extraction permet de déterminer 3 fractions distinctes, évoluant indépendamment et de compositions et de structures différentes, pouvant se former selon le mécanisme suivant:

- dès la mise en contact du lait avec la membrane, se forme la couche superficielle (notée ici SR), très faiblement fixée, issue de la couche de polarisation. Elle constitue le colmatage réversible, dont l'épaisseur est éminemment fonction des conditions opératoires : durée en statique, plus vitesse de circulation et pression en dynamique. De composition protéique analogue à celle du lait, elle participe, en s'épaississant, à la diminution du débit d'ultrafiltrat;

- alimentée par la précédente, apparaît, elle aussi très rapidement, la couche notée ici US; plus compacte, elle jouerait le rôle de membrane ultrafiltrante. Adsorbée à la surface de la membrane, elle constitue le colmatage irréversible car inextractible à l'eau sauf avec des ultrasons;

- formée pendant l'apparition des précédentes, la couche notée ici RM, adsorbée dans la membrane, correspond au colmatage interne, faible et variant peu en masse avec les conditions opératoires.

Le processus d'apparition de l'ensemble du dépôt paraît donc achevé dès les premières minutes. Par la suite, les évolutions sont surtout quantitatives.

La création d'un champ électrique transmembranaire permet d'intervenir sur ce colmatage et de le diminuer, surtout au niveau de la couche réversible, et entraîne une augmentation du débit d'ultrafiltrat qui peut atteindre $50 \%$ : c'est donc une technique intéressante.

L'application de cette technique au niveau industriel, malgré des difficultés de réalisation pour l'établissement d'un champ électrique, peut apporter des améliorations sensibles dans le traitement de fluides biologiques dont le rétentat ou le filtrat présenterait une forte valeur ajoutée.

II reste à optimiser le procédé d'électroultrafiltration par la recherche des conditions les meilleures et par un montage sur pilote permettant d'augmenter l'efficacité du champ tout en limitant l'électrolyse.

Parallèlement, la poursuite de l'étude en statique et en dynamique dans des condi- 
tions améliorées (mode opératoire et analyses) devrait nous permettre d'affiner notre connaissance du mécanisme de formation du dépôt et de l'action du champ électrique.

\section{RÉFÉRENCES}

Ahlgreen RM (1980) Electrodialysis and ultrafiltration as a combined process. In: Polymer Science and Technology. Vol 13: Ultrafiltration Membranes and Applications (AR Cooper, ed) Plenum Press, New York - Londres, 609-618

Alais C (1984) Science du lait. Principe des techniques laitières. SEPAIC, Paris

Baumy JJ, Brulé G (1988a) Binding of bivalent cations to $\alpha$-lactalbumin and $\beta$-lactoglobulin: effect of $\mathrm{pH}$ and ionic strength. Lait $68,33-48$

Baumy JJ, Brulé G (1988b) Effect of $\mathrm{pH}$ and ionic strength on the binding of bivalent cations to $\beta$-casein. Lait $68,409-417$

Bennasar M (1984) Étude de l'ultrafiltration sur membranes minérales. Application au lait. Thèse, Montpellier

Brulé G, Fauquant J (1981) Mineral balance in skim-milk and milk retentate: effect of physicochemical characteristics of the aqueous phase. J Dairy Res 48, 91-97

Cheryan M, Merin U (1980) A study of the fouling phenomenon during ultrafiltration of cottage cheese whey. In: Polymer Science and Technology. Vol 13. Ultrafiltration Membranes and Applications (AR Cooper, ed) Plenum Press, New York - Londres, 619-629

Cohen T, Doyen JJ, Dagard P, Letord MM, Gavach C, Brunea J (1988) Nouvelles membranes perfluorées pour la récupération et la reconcentration des acides par des procédés électromembranaires. Filtra 88 , Société Française de Filtration, Paris, 66-81

Escudier JL, Moutounet M, Cottereau P (1988) Applications des techniques à membranes aux produits de la vigne : exemple de l'électrodialyse. Filtra 88 , Société Française de Filtration, Paris, 329-335

Fox PF, Mulvihill DM (1982) Milk protein: molecular, colloidal and functional properties. J Dairy Res 49, 679-693
Freund P (1989) Étude des phénomènes de transport, en ultrafiltration et électroultrafiltration sur membranes minérales, de solutions protéiques modèles. Thèse, Montpellier

Gernedel C (1980) Über die Ultrafiltration von Milch und die den Widerstand der Ablagerungsschicht beeinflussenden Faktoren. Thèse, Munich

Green ML (1982a) Effect on the composition and properties of casein micelles of interaction with ionic materials. J Dairy Res 49, 8798

Green ML (1982b) Mode of binding of ionic materials to casein micelles. J Dairy Res $49,99-$ 105

Guizard C, Légault F, Idrissi N, Larbot A, Cot L, Gavach C (1989) Electronically conductive mineral membranes designed for electroultrafiltration. J Membr Sci 41, 127-142

Helfferich $F$ (1962) lon exchange. McGraw-Hill Book Company Inc, New York

Holt C (1982) Inorganic constituents of milk. III. The colloidal calcium phosphate of cow's milk. J Dairy Res 49, 29-38

Kimura S, Tamano A (1986) Separation of amino-acids by charged ultrafiltration membranes. Membr Membr Processes 191-197

Krishnaswamy P, Klinkovski P (1986) Electrokinetics and electrofiltration. Adv Solid-liq Sep 291-319

Letord MM (1986) Électrodialyse et électroélectrodialyse. Bilan d'utilisation en France. Filtra 86 , Société Française de Filtration, Paris, 356-369

Milisic V, Bersillon JL (1986) Antifouling techniques in cross-flow ultrafiltration. Filtr Sep 23, 347-349

Radovitch JM, Sparks RE (1980) Electrophoretic techniques for controlling concentration polarization in ultrafiltration. In: Polymer Science and Technology. Vol 13. Ultrafiltration Membranes and Applications (AR Cooper, ed) Plenum Press, New York-Londres, 249-268

Rios G, Rakotoarisoa H, Tarodo de la Fuente B, Rumeau M (1986) Synergisme obtenu par couplage d'un champ électrique ou d'un lit fluidisé avec une membrane d'ultrafiltration. Filtra 86 , Société Française de Filtration, Paris, 380-394 
Schmidt DG (1982) Association of caseins and casein micelle structure. In: Developments in Dairy Chemistry. 1. Proteins (Fox PF, ed) Applied Science Publishers, Londres, 61-86

Swaisgood HE (1982) Chemistry of milk protein. In: Developments in Dairy chemistry. 1. Proteins (Fox PF, ed) Applied Science Publishers, Londres, 1-59

Taddei C, Aimar P, Daufin G, Sanchez V (1988) Factors affecting fouling of an inorganic membrane during sweet whey ultrafiltration. Lait 68, 157-176

Takahashi S, Nakayasu K (1987) Recovery of amino-acids from amino-acid alkaline salts. Brevet japonais JP 6230742 (8730 742) (9/ 2/87)

Tarodo de la Fuente $B$, Teissèdre PL, Freund $P$, Bennasar M (1989) Essais de nouvelles membranes minérales d'ultrafiltration pour l'industrie alimentaire. Partie 1 : Application au lait écrémé et caillé lactique. Ind Aliment Agric 106, 287-292

Turkson AK (1987) Electrofiltration of bovine serumalbumine with rotating dynamic mem- branes. Filtech Conf Utrecht 23/9/1987, The Filtration Society, Leics, 294-301

Vallot D (1988) Procédé et appareils industriels de déminéralisation en continu par le courant électrique. Filtra 88 , Société Française de Filtration, Paris, 90-109

Vétier C, Bennasar M, Tarodo de la Fuente B (1986) Étude des interactions entre constituants du lait et membranes minérales de microfiltration. Lait 66, 269-287

Visvanathan C, Ben Aïm R (1986) L'application d'un champ électrique en microfiltration tangentielle pour éviter le colmatage interne des pores de la membrane. Filtra 86 , Société Française de Filtration, Paris, 334-341

Vivoni D, Aimar P, Sanchez V (1986) Modification de la polarisation de concentration en ultrafiltration par l'action d'un champ électrique. Filtra 86 , Société Française de Filtration, Paris, 321-333

Wakeman R (1986) Electrofiltration $=$ Microfiltration + Electrophoresis. Chem Eng 426, 65-70

Zidoune MN (1983) Étude de l'ultrafiltration des lactosérums sur membranes minérales. Thèse, Montpellier 